\title{
Ion Channel Stochasticity May Be Critical in Determining the Reliability and Precision of Spike Timing
}

\author{
Elad Schneidman \\ Department of Neurobiology, Institute of Life Sciences, Institute of Computer Science, \\ and Center for Neural Computation, Hebrew University, Jerusalem 91904, Israel
}

Barry Freedman

Idan Segev

Department of Neurobiology, Institute of Life Science and Center for Neural Computation, Hebrew University, Jerusalem 91904, Israel

The firing reliability and precision of an isopotential membrane patch consisting of a realistically large number of ion channels is investigated using a stochastic Hodgkin-Huxley $(\mathrm{HH})$ model. In sharp contrast to the deterministic $\mathrm{HH}$ model, the biophysically inspired stochastic model reproduces qualitatively the different reliability and precision characteristics of spike firing in response to DC and fluctuating current input in neocortical neurons, as reported by Mainen \& Sejnowski (1995). For DC inputs, spike timing is highly unreliable; the reliability and precision are significantly increased for fluctuating current input. This behavior is critically determined by the relatively small number of excitable channels that are opened near threshold for spike firing rather than by the total number of channels that exist in the membrane patch. Channel fluctuations, together with the inherent bistability in the $\mathrm{HH}$ equations, give rise to three additional experimentally observed phenomena: subthreshold oscillations in the membrane voltage for DC input, "spontaneous" spikes for subthreshold inputs, and "missing" spikes for suprathreshold inputs. We suggest that the noise inherent in the operation of ion channels enables neurons to act as "smart" encoders. Slowly varying, uncorrelated inputs are coded with low reliability and accuracy and, hence, the information about such inputs is encoded almost exclusively by the spike rate. On the other hand, correlated presynaptic activity produces sharp fluctuations in the input to the postsynaptic cell, which are then encoded with high reliability and accuracy. In this case, information about the input exists in the exact timing of the spikes. We conclude that channel stochasticity should be considered in realistic models of neurons. 


\section{Introduction}

Following the formulation of the Hodgkin-Huxley $(\mathrm{HH})$ equations for modeling spike initiation in the squid giant axon (Hodgkin \& Huxley, 1952), research into the electrical activity of single neurons followed two main paths: the attempt to discover further macroscopic equations governing different membrane currents (e.g., Yamada, Koch, \& Adams, 1989), and the attempt to investigate, and mathematically describe, the behavior of the ion channels underlying these currents (see Hille, 1992; Sakmann \& Neher, 1995). Although part of the same general problem, mathematically these two areas of investigation are entirely different. In the $\mathrm{HH}$ formulation, the ion conductances are modeled by means of deterministic differential equations, and their values range continuously from zero to a given maximum. However, because individual ion channels are discrete elements whose properties can be given only probabilistically, the electrical activity of nerve cells is most accurately described as resulting from the interaction of stochastic, discrete units. It is commonly assumed that a large collection of such discrete units practically forms a continuous deterministic system, as is the case in numerous large physical systems. Because the number of excitable channels in the axon's spike initiation zone is estimated to be large (on the order of tens of thousands of ion channels; Hille, 1992), models for spike generation in neurons typically use deterministic rather than stochastic equations (Mainen, Joerges, Huguenard, \& Sejnowski, 1995; Rinzel \& Ermentrout, 1989; Rapp, Yarom, \& Segev, 1996).

A few theoretical studies did consider the effect of channel stochasticity, focusing on the question, "When does the stochastic model converge to the corresponding deterministic model?" The pioneering work of Fitzhugh (1965) used a kinetic (stochastic) model for the conductance change associated with the $\mathrm{HH}$ equations; others used stochastic $\mathrm{HH}$ equations to investigate the effect of various parameters (e.g., number of channels, membrane area) on the dynamics of the membrane voltage. The main message of these studies is that the stochastic system differs considerably from the deterministic $\mathrm{HH}$ system when a small number (a few hundred) of channels and small membrane areas are involved (Skaugen \& Walløe, 1979; Strassberg \& DeFelice, 1993; DeFelice \& Isaac, 1992). Other aspects of this problemspontaneous spiking due to channel noise and the effect of channel stochasticity on spike propagation in axons-were recently explored (Rubinstein, 1995; Chow \& White, 1996; Horikawa, 1991, 1993). In a more general perspective, the effect of different kinds of noise on the firing threshold of neurons was examined by Lecar and Nossal $(1971 a, b)$. Recently Jensen and Gartner (1997) have dealt with the effect of additive white noise on the firing reliability of different neuron models (see also Longtin \& Hinzer, 1996, and Braun, Huber, Dewald, Schafer, \& Voigt, 1998).

A recent experimental study by Mainen and Sejnowski (1995) on the reliability of spike firing time in neocortical pyramidal cells motivated us 
to readdress the question, "How good an approximation is the deterministic model, given a large number of excitable channels?" focusing on the timing of the spikes during the train. Mainen and Sejnowski showed that spike timing is highly unreliable for repeated DC current inputs, whereas fluctuating current inputs significantly improve the firing reliability and the firing precision up to a millisecond range (see also the recent studies by Nowak, Sanches-Vives, \& McCormick, 1997 and Tang, Bartels, \& Sejnowski, 1997 and studies on the reliability of neuronal spike firing times in behaving animals, e.g., de Ruyter van Steveninck, Lewen, Strong, Koberle \& Bialek, 1997, and Reich, Victor, Knight, Ozaki, \& Kaplan, 1997). We were interested in exploring if, for realistic membrane area and number of excitable channels, a biophysically inspired noise that is generated by channel stochasticity plays an important role in determining the reliability of spike firing times in the spike initiation zone. In this context, it is noteworthy that channel noise was shown to be significant for the macroscopic behavior of neurons (Volgushev, Chistiakova, \& Singer, 1998), and in other excitable membranes (Berzukov \& Vodyanoy, 1995). We therefore modeled membrane patches of areas of a few hundred square micrometers, comprising a total of a few tens of thousands ion channels that receive both DC inputs and the more biologically realistic fluctuating current inputs. We show that for a broad range of inputs, the stochastic equations generate results that are strikingly different from those obtained from the corresponding deterministic $\mathrm{HH}$ equations. In addition to its significant effect on the timing of spike firing, channel noise also produces three additional experimental observations: voltage-dependent subthreshold membrane voltage oscillations for DC input, occasional "missing" spikes for suprathreshold inputs and "spontaneous" spikes for subthreshold inputs. In section 4, we speculate on the functional implications of channel stochasticity for neural coding. A preliminary account of this work has appeared as an abstract (Schneidman, Freedman, \& Segev, 1997).

\section{Basic Model and Simulation Scheme}

The membrane dynamics of the $\mathrm{HH}$ equations is given by

$$
C_{m} \frac{d V}{d t}=-g_{L}\left(V-V_{L}\right)-g_{K}\left(V-V_{K}\right)-g_{N a}\left(V-V_{N a}\right)+I,
$$

where $V$ is the membrane potential; $V_{L}, V_{K}, V_{N a}$ are the reversal potentials of the leakage, potassium, and sodium currents, respectively. $g_{L}, g_{K}, g_{\mathrm{Na}}$, are the corresponding specific ion conductances; $C_{m}$ is the specific membrane capacitance; and $I$ is the specific current injected into this membrane patch. The voltage-dependent conductances for the potassium and sodium channels are given by

$$
g_{K}(V, t)=\bar{g}_{K} n^{4} ; \quad g_{N a}(V, t)=\bar{g}_{N a} m^{3} h,
$$


Table 1: Hodgkin-Huxley Parameters and Rate Functions Used in the Simulations.

\begin{tabular}{cll}
\hline & & \\
$C_{m}$ & Specific membrane capacitance & $1 \mu \mathrm{F} / \mathrm{cm}^{2}$ \\
$T$ & Temperature & $6.3^{\circ} \mathrm{C}$ \\
$V_{L}$ & Leakage reversal potential & $10.6 \mathrm{mV}$ \\
$g_{L}$ & Leakage conductance & $0.3 \mathrm{mS} / \mathrm{cm}^{2}$ \\
$V_{K}$ & Potassium reversal potential & $-12 \mathrm{mV}$ \\
$\bar{g}_{K}$ & Maximal potassium conductance & $36 \mathrm{mS} / \mathrm{cm}^{2}$ \\
$\gamma_{K}$ & Potassium channel conductance & $20 \mathrm{pS}$ \\
$D_{K}$ & Potassium ion channel density & $18 \mathrm{channels} / \mu \mathrm{m}^{2}$ \\
$V_{N a}$ & Sodium reversal potential & $115 \mathrm{mV}$ \\
$\bar{g}_{N a}$ & Maximal sodium conductance & $120 \mathrm{mS} / \mathrm{cm}^{2}$ \\
$\gamma_{N a}$ & Sodium channel conductance & $20 \mathrm{pS}$ \\
$D_{N a}$ & Sodium ion channel density & $60 \mathrm{channels} / \mu \mathrm{m}^{2}$ \\
& & \\
& & $0.01(10-V)$ \\
$\alpha_{n}(V)$ & & $\exp (10-V) / 10]-1$ \\
$\alpha_{m}(V)$ & & $0.1(25-V)$ \\
$\alpha_{h}(V)$ & & $0.07 \exp [-V / 20]-1$ \\
$\beta_{n}(V)$ & & $0.125 \exp [-V / 80]$ \\
$\beta_{m}(V)$ & & $4.0 \exp [-V / 18]$ \\
$\beta_{h}(V)$ & & $\frac{1}{\exp [(30-V) / 10]+1}$ \\
& & \\
\hline
\end{tabular}

where the dynamics of $n$ (and similarly for $m$ and $h$ ) is given by

$$
\frac{d n}{d t}=\alpha_{n}(1-n)-\beta_{n} n
$$

The rate functions $\alpha^{\prime}$ s, $\beta^{\prime}$ s, and the maximal conductances $\bar{g}$ 's, as formulated by Hodgkin and Huxley, are given in Table 1.

Equations 2.1 through 2.3 give an extremely successful description of the mean behavior of the voltage and currents in the squid giant axon, without the need to treat the activity of individual ion channels that underlie this behavior. Hodgkin and Huxley suggested a physical interpretation of their equations-namely, that the term $n^{4}$ indicates that there are four separate gates and that a $\mathrm{K}^{+}$channel is opened only when all these gates are open. The term $m^{3} h$ indicates that there are three $m$-gates and one $h$-gate that must be open to enable the $\mathrm{Na}^{+}$current to flow via the sodium channel. A corresponding kinetic model (Fitzhugh, 1965; Clay \& DeFelice, 1983) can be formalized as a Markov model that explicitly incorporates the internal workings of the ion channels. The model, which is equivalent in the limit of large number of channels to the HH model (Skaugen \& Walløe, 1979), suggests that the $\mathrm{K}^{+}$channel can exist in five different states and that the kinetic scheme describing the behavior of this channel is given by

$$
\left[\mathbf{n}_{\mathbf{0}}\right] \underset{\beta_{n}}{\stackrel{4 \alpha_{n}}{\rightleftharpoons}}\left[\mathbf{n}_{\mathbf{1}}\right] \underset{2 \beta_{n}}{\stackrel{3 \alpha_{n}}{\rightleftharpoons}}\left[\mathbf{n}_{\mathbf{2}}\right] \underset{3 \beta_{n}}{\stackrel{2 \alpha_{n}}{\rightleftharpoons}}\left[\mathbf{n}_{\mathbf{3}}\right] \underset{4 \beta_{n}}{\stackrel{\alpha_{n}}{\rightleftharpoons}}\left[\mathbf{n}_{\mathbf{4}}\right]
$$


where $\left[\mathbf{n}_{\mathbf{i}}\right]$ is the number of the channels with $i$ open gates and, hence, $\left[\mathbf{n}_{\mathbf{4}}\right]$ labels the single open state of the $\mathrm{K}^{+}$channel. $\alpha_{n}, \beta_{n}$ are identical to the original $\mathrm{HH}$ rate functions.

Similarly, in this description, each $\mathrm{Na}^{+}$channel can exist in eight different states, as in the following scheme,

$$
\begin{aligned}
& {\left[\mathbf{m}_{\mathbf{0}} \mathbf{h}_{\mathbf{1}}\right] \underset{\beta_{m}}{\stackrel{3 \alpha_{m}}{\rightleftharpoons}}\left[\mathbf{m}_{\mathbf{1}} \mathbf{h}_{\mathbf{1}}\right] \underset{2 \beta_{m}}{\stackrel{2 \alpha_{m}}{\rightleftharpoons}}\left[[ \mathbf { m } _ { \mathbf { 2 } } \mathbf { h } _ { \mathbf { 1 } } ] \underset { 3 \beta _ { m } } { \stackrel { \alpha _ { m } } { \rightleftharpoons } } \left[\left[\mathbf{m}_{\mathbf{3}} \mathbf{h}_{\mathbf{1}}\right]\right.\right.} \\
& \alpha_{h} \uparrow \downarrow \beta_{h} \quad \alpha_{h} \uparrow \downarrow \beta_{h} \quad \alpha_{h} \uparrow \downarrow \beta_{h} \quad \alpha_{h} \uparrow \downarrow \beta_{h} \\
& {\left[\mathbf{m}_{\mathbf{0}} \mathbf{h}_{\mathbf{0}}\right] \underset{\beta_{m}}{\stackrel{3 \alpha_{m}}{\rightleftharpoons}}\left[\mathbf{m}_{\mathbf{1}} \mathbf{h}_{\mathbf{0}}\right] \underset{2 \beta_{m}}{\stackrel{2 \alpha_{m}}{\rightleftharpoons}}\left[\mathbf{m}_{\mathbf{2}} \mathbf{h}_{\mathbf{0}}\right] \underset{3 \beta_{m}}{\stackrel{\alpha_{m}}{\rightleftarrows}}\left[\mathbf{m}_{\mathbf{3}} \mathbf{h}_{\mathbf{0}}\right],}
\end{aligned}
$$

where $\left[\mathbf{m}_{\mathbf{i}} \mathbf{h}_{\mathbf{j}}\right]$ refers to the number of channels within the population that are currently in the state $m_{i} h_{j},\left[\mathbf{m}_{3} \mathbf{h}_{\mathbf{1}}\right]$ labels the single open state of the $\mathrm{Na}^{+}$ channel, and $\alpha_{h}, \beta_{h}, \alpha_{m}$, and $\beta_{m}$ are the rate functions in $\mathrm{HH}$ formalism. The potassium and sodium membrane conductances are given by

$$
g_{K}(V, t)=\gamma_{K}\left[\mathbf{n}_{4}\right] ; \quad g_{N a}(V, t)=\gamma_{N a}\left[\mathbf{m}_{3} \mathbf{h}_{\mathbf{1}}\right],
$$

where $\gamma_{\mathrm{K}}$ and $\gamma_{\mathrm{Na}}$ are the conductances of the single potassium and sodium ion channel at their open state, respectively.

By switching from the standard HH model to Fitzhugh's (1965) model, channel stochasticity is incorporated into the voltage dynamics. Instead of keeping track of each of the channels separately, we have used a more efficient scheme to track only the total populations of channels in each of their possible states (see Skaugen \& Walløe, 1979, and Chow \& White, 1996, for a discussion of possible simulation methods for populations of channels). Specifically, if at time $t$ there are $n_{A}$ channels in state $A$ and $n_{B}$ channels in state $B$ and the transfer rate of channels from state $A$ to state $B$ is $r$, then each of the channels in state $A$ might transfer to state $B$ between time $t$ and $t+\Delta t$ with probability $p=r \Delta t$. Hence, for each time step, we determine $\Delta n_{A B}$, the number of channels that move from $A$ to $B$, by choosing a random number from a binomial distribution (Press, Teukolsky, Vetterling, \& Flannery, 1992),

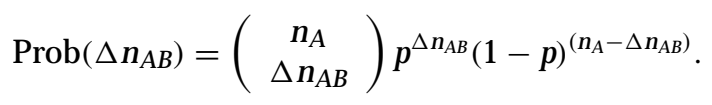

In this study, we used the forward Euler integration method with $\Delta t=$ $0.01 \mathrm{msec}$, as in Chow and White (1996). It is important to note that the spatially independent (space-clamped) HH equations were utilized; we simulate isopotential membrane patches of varying areas. Clearly, this is a severe oversimplification of the realistic case, and its implications will be addressed in section 4. Finally, in order to make the transition from the deterministic to the stochastic model, we need to know how many channels there are in the modeled membrane patch. Once we choose the conductance of the 
individual channel, the number of channels can be calculated directly from the channel densities and from the maximal conductances, $\bar{g}$ 's, given in the HH model (see Table 1).

\section{Results}

Before we proceed to the actual simulation results, we first try to estimate the effect of introducing stochasticity into the $\mathrm{HH}$ model. Suppose that the area of the membrane patch is $200 \mu^{2}$. With the parameters of Table 1, this membrane patch bears $3600 \mathrm{~K}^{+}$channels and $12,000 \mathrm{Na}^{+}$channels. Considering the large number of modeled channels, one would naively estimate the number of fluctuating channels about the mean to be on the order of $\frac{\sqrt{N}}{N}$ channels. Hence, for $N=3600 \mathrm{~K}^{+}$channels, the size of the fluctuation is $1.7 \%$, and we would expect rather small deviations from the deterministic model. An even smaller effect would be expected for the $\mathrm{Na}^{+}$ channels. Surprisingly, this is not the case, as shown in Figure 1.

3.1 Encoding Reliability and Precision: Input Current Versus Channel Fluctuations. The response of a stochastic isopotential HH compartment to repeated presentation of suprathreshold currents is shown in Figure 1. When the same suprathreshold DC current pulse $\left(10 \mu \mathrm{A} / \mathrm{cm}^{2}, 250 \mathrm{msec} ; \mathrm{A}\right.$, top frame) is repeatedly presented to the modeled membrane patch, the resulting spike trains vary considerably from trial to trial; the spike firing time is neither reliable nor accurate (see Figure 1A, bottom frame). This should be compared with the response of the corresponding deterministic model shown in the middle frames. In contrast, when the stimulus is fluctuating (simulating the current that presumably reaches the site of spike generation following the activation of many synaptic inputs impinging on the dendritic tree, B top frame; see the caption of Figure 1 for details), the reliability and accuracy of the spike train in the stochastic $\mathrm{HH}$ model are improved compared to DC case (see Figure 1B, bottom frame).

As in the study of Mainen and Sejnowski (1995), two measures of the spike timing, the reliability and the precision, were calculated from the peristimulus time histogram (PSTH, not shown) for a wide range of input patterns (see caption of Figure 2 for details). The reliability and precision of the spike patterns were strongly correlated with the amplitude of the fluctuations in the input current, $\sigma_{\text {input }}$ (see Figures 2A and 2B); the reliability and precision dropped as the input was filtered with larger time constants (see Figures 2C and 2D). In the stochastic $\mathrm{HH}$ model, both the reliability and the precision, which for most of the responses was in the range of 1 to $2 \mathrm{msec}$, are in close agreement with the results of Mainen and Sejnowski (1995). It is noteworthy that there is no clear dependence of the reliability and precision on the mean value of the injected current, as was also found experimentally by Mainen and Sejnowski (personal communication). 

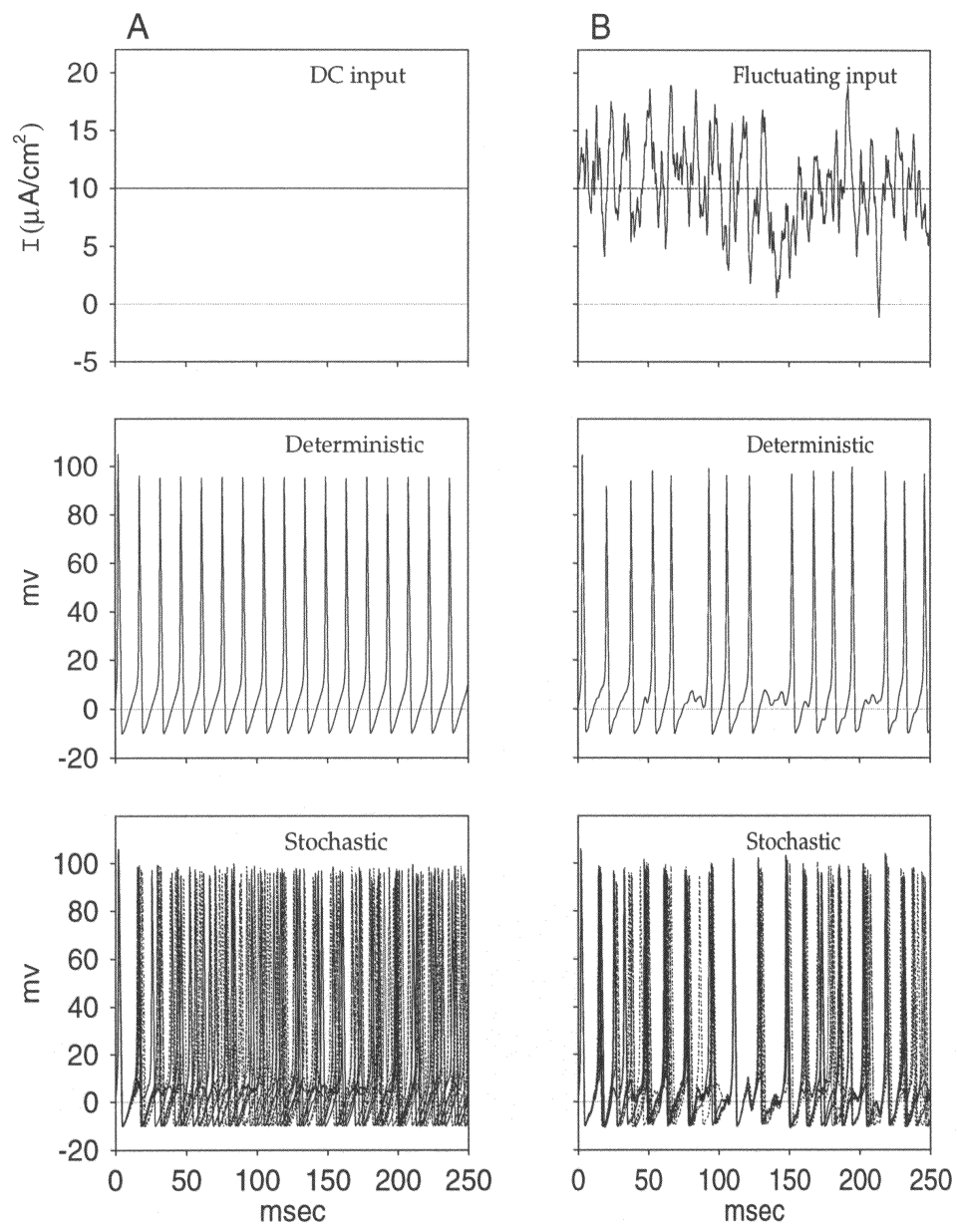

Figure 1: Reliability of firing patterns in a model of an isopotential HodgkinHuxley membrane patch in response to both DC and fluctuating current input. (A) Ten superimposed responses to repeated suprathreshold DC current pulses $\left(10 \mu \mathrm{A} / \mathrm{cm}^{2}, 250 \mathrm{msec}\right.$; top frame) evoked a train of regular firing in the deterministic $\mathrm{HH}$ model (middle frame) and a jitter in the firing in the stochastic $\mathrm{HH}$ model (bottom frame). (B) The same patch was again stimulated 10 times repeatedly, this time with a fluctuating stimulus (low-pass gaussian white noise with a mean $\bar{I}$, of $10 \mu \mathrm{A} / \mathrm{cm}^{2}$, and a standard deviation $\sigma_{\text {input }}$ of $7 \mu \mathrm{A} / \mathrm{cm}^{2}$, which was convolved with an alpha function with a time constant $\tau_{\text {input }}=1 \mathrm{msec}$, top frame; see Mainen \& Sejnowski, 1995). As can be clearly seen, the jitter in spike timing in the stochastic model is significantly smaller in B than in A (i.e., increased accuracy for the fluctuating current input). Patch area used was $200 \mu \mathrm{m}^{2}$, with $3,600 \mathrm{~K}^{+}$channels and $12,000 \mathrm{Na}^{+}$channels. (Compare to Figure 1 in Mainen \& Sejnowski, 1995.) 

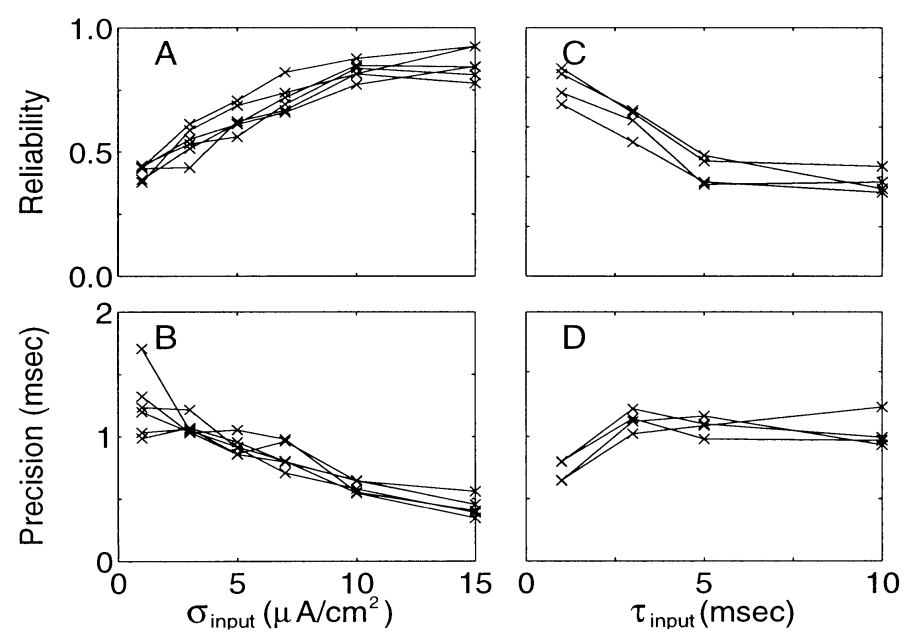

Figure 2: Dependence of reliability and precision on stimulus parameters. The reliability and accuracy of the spike train was calculated in a similar manner to that of Mainen \& Sejnowski (1995). The peristimulus time histogram (PSTH) of 20 successive presentations of a particular stimulus was smoothed using an adaptive filter, yielding an estimate for the instantaneous firing rate. Significant elevations in the instantaneous firing rate ("events") were selected from the PSTH using a threshold of two times the mean firing rate over a given block of responses. The reliability of the response to a particular stimulus is defined as the average of the fraction of spikes that occurred in the events in that stimulus' PSTH. The temporal precision of the response is defined by the average of standard deviation of spike timing within the events in that stimulus's PSTH. (A) Estimates of the reliability of the spike train in a $200 \mu \mathrm{m}^{2}$ stochastic $\mathrm{HH}$ membrane patch, for stimuli with various fluctuation amplitudes, $\sigma_{\text {input }}$. Each curve is for a different mean value of the stimulus $\left(\bar{I}=7-20 \mu \mathrm{A} / \mathrm{cm}^{2}, \tau_{\text {input }}=\right.$ $1 \mathrm{msec}$ ). (B) The temporal precision of the same responses as in A. (C) The reliability for stimuli filtered with different time constants $\left(\tau_{\text {input }}=1-10 \mathrm{msec}\right)$. Each curve is for a different mean value of the stimulus and a given $\sigma_{\text {input }}$. $\left(\bar{I}=7-20 \mu \mathrm{A} / \mathrm{cm}^{2}, \sigma_{\text {input }}=3-12 \mu \mathrm{A} / \mathrm{cm}^{2}\right)$. (D) The temporal precision of the same responses as in $\mathrm{C}$.

Hence, with a realistically large number of channels, when incorporating their unavoidable stochasticity, one obtains an effect that is qualitatively similar to the behavior of real neurons and is significant from both biophysical and computational viewpoints. Clearly, the effect of stochasticity depends on the number of ion channels and the membrane area. It increases 
when decreasing the number of channels and decreases when increasing the membrane area. Still, the effect of channel stochasticity was significant even when the membrane area was increased by a factor of five (to $1000 \mu \mathrm{m}^{2}$ ) as well as when, for a given membrane patch, the channel density was increased by the same factor (not shown; see section 4). But why is the result of the stochastic model so different from that obtained from the corresponding deterministic HH model?

The apparent error in the previous estimation (see the beginning of section 3) of the size of the effect of channel stochasticity lies in failing to realize that the relevant number of channels is not the total number of channels in the membrane patch, but rather the number of channels that are open near the threshold for spike firing. If this number is relatively small, the size of the fluctuations in the number of open channels in this regime is not negligible. Mathematically, the correct estimation for the size of the fluctuation should rely on the binomial statistics. For a total population of $N$ channels and a probability $p$ of a channel to be open, the size of the fluctuations is $\sqrt{N p(1-p)}$ and the fluctuation relative to the mean, $N p$, is $\sqrt{\frac{(1-p)}{N p}}$. If $p$ is small, as in the case of near threshold for spike firing, the relative size of the fluctuations is rather large. In this case, the inherent stochasticity of the channels is expected to have a significant effect on the voltage dynamics and, specifically, on the time of threshold crossing. When this is the case, the firing behavior of the stochastic model is expected to be considerably different from that of the corresponding deterministic model.

Figure 3 shows that this is indeed the situation. As in Figure 1A, 10 repeated $10 \mu \mathrm{A} / \mathrm{cm}^{2} \mathrm{DC}$ current inputs were applied, this time to a $600 \mu \mathrm{m}^{2}$ membrane patch consisting of a total of $10,800 \mathrm{~K}^{+}$channels and $36,000 \mathrm{Na}^{+}$ channels. The voltage response is shown in Figure 3B, whereas the numbers of open $\mathrm{K}^{+}$and $\mathrm{Na}^{+}$channels near the threshold for spike firing are shown in panels $C$ and D, respectively. A surprisingly small number of ion channelsapproximately $300 \mathrm{~K}^{+}$channels and $50 \mathrm{Na}^{+}$channels-are opened in this voltage regime. With these small numbers, channel fluctuations become significant and critically determine the exact time in which a sufficient number of additional $\mathrm{Na}^{+}$channels is recruited to initiate a regenerative response. When injecting the same DC current repeatedly, the fluctuations vary significantly from one trial to the other, and, consequently, the time of spike firing for this input is unreliable.

In principle, this channel-noise-induced unreliability can be mostly overridden by injecting a current that fluctuates significantly. If the input fluctuations are sufficiently large, the voltage dynamics will be dominated by the transients in the current input rather than by the channel noise. This effect is demonstrated in Figure 4, where the response to a fluctuating input in a specific time window is shown. The current input is depicted in $A$, the voltage response is shown in panel $\mathrm{B}$, and the number of open $\mathrm{K}^{+}$and $\mathrm{Na}^{+}$ channels is shown in panels $C$ and $D$, respectively. In contrast to the DC- 

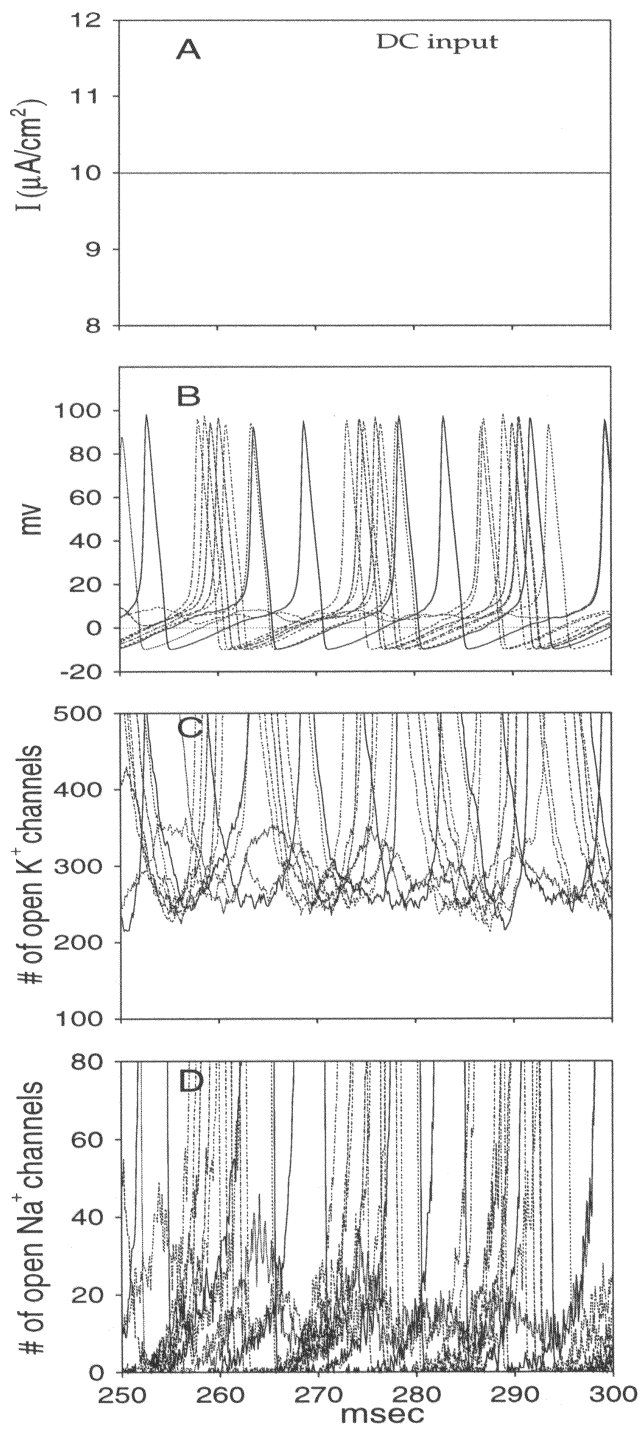

Figure 3: Channel fluctuations ruin the reliability of spike timing in the case of DC current input. (A) $10 \mu \mathrm{A} / \mathrm{cm}^{2} \mathrm{DC}$ current injected to a $600 \mu \mathrm{m}^{2}$ stochastic $\mathrm{HH}$ model $\left(10,800 \mathrm{~K}^{+}\right.$channels and $36,000 \mathrm{Na}^{+}$channels) results with dispersed spike timings for repeated simulation ( 10 superimposed voltage traces) in B. (C, D) the number of open $\mathrm{Na}^{+}$and $\mathrm{K}^{+}$channels, respectively, corresponding to the voltage traces presented in $\mathrm{B}$. 
input case (see Figure 3), here the transients in the input current partially overcome the channel fluctuations and enforce 9 out of the 10 spikes to occur within an approximately $1 \mathrm{msec}$ time window (see Figure 4B). The reason for this relatively high reliability of spike timing becomes clear by observing panels $C$ and $D$. The accuracy is determined by two parameters. The first is the variability in the time where the number of open $\mathrm{K}^{+}$channels reaches a sufficiently small value (note that a large outward $\mathrm{K}^{+}$current impedes the initiation of the spike). This variability should be small in order to obtain high accuracy. Indeed, in 9 of 10 repetitions, this condition is satisfied (see Figure 4C). The second parameter is the rate of the buildup of the $\mathrm{Na}^{+}$channel population toward threshold. For an accurate spike timing, this buildup, which is determined by the amplitude and rate of the depolarizing input current, should be sufficiently large to overcome the channel fluctuations (see Figure 4D). For a given voltage, the size of the channel fluctuation is as large in the fluctuating-input case as in the DC-input case, but in the former these channels fluctuations are "lost in the crowd."

To examine the relative contribution of the $\mathrm{K}^{+}$and $\mathrm{Na}^{+}$channels to the reliability and precision, we simulated a hybrid system in which one of the channel populations was stochastic and the other was deterministic. Both channel types contribute to the complex reliability nature of the system. However, as expected from the larger number of $\mathrm{K}^{+}$channels that are open near threshold for spike firing (see Figures $3 \mathrm{C}$ and $3 \mathrm{D}$ and Figures $4 \mathrm{C}$ and $4 \mathrm{D})$, as well as from their slower kinetics, the noise introduced by the $\mathrm{K}^{+}$ channels is more dominant in determining the reliability and accuracy of this system (not shown).

3.2 Subhreshold Oscillations, "Spontaneous" Spikes, and "Missing" Spikes. Along with the effect of channel fluctuations on spike timing, incorporating channel stochasticity in the $\mathrm{HH}$ model gives rise to three additional phenomena that were observed experimentally: (1) considerable subthreshold oscillations in the membrane voltage for DC inputs, (2) "spontaneous" spikes for "subthreshold" inputs, and (3) "missing" spikes for suprathreshold inputs. These phenomena cannot be reproduced in the deterministic $\mathrm{HH}$ model. In the stochastic model, oscillations in the membrane voltage are already observed for zero current input (see Figure 5A). Occasionally these oscillations are sufficiently large to generate "spontaneous" spikes, which would not have occurred in the corresponding deterministic model (current threshold for spike firing in the deterministic model is $\left.I=7 \mu \mathrm{A} / \mathrm{cm}^{2}\right)$. An example of "spontaneous" spikes in the case of $I=4 \mu \mathrm{A} / \mathrm{cm}^{2}$ is shown in Figure 5B; detailed analysis of spontaneous spiking in the stochastic $\mathrm{HH}$ model for zero current input was recently performed by Chow and White (1996). Compared to the deterministic model, where regular repetitive firing occurs (see Figure 1), "missing" spikes (with respect to the corresponding deterministic model) are observed in the stochastic model for suprathreshold currents (see Figures 5C and 5D). 

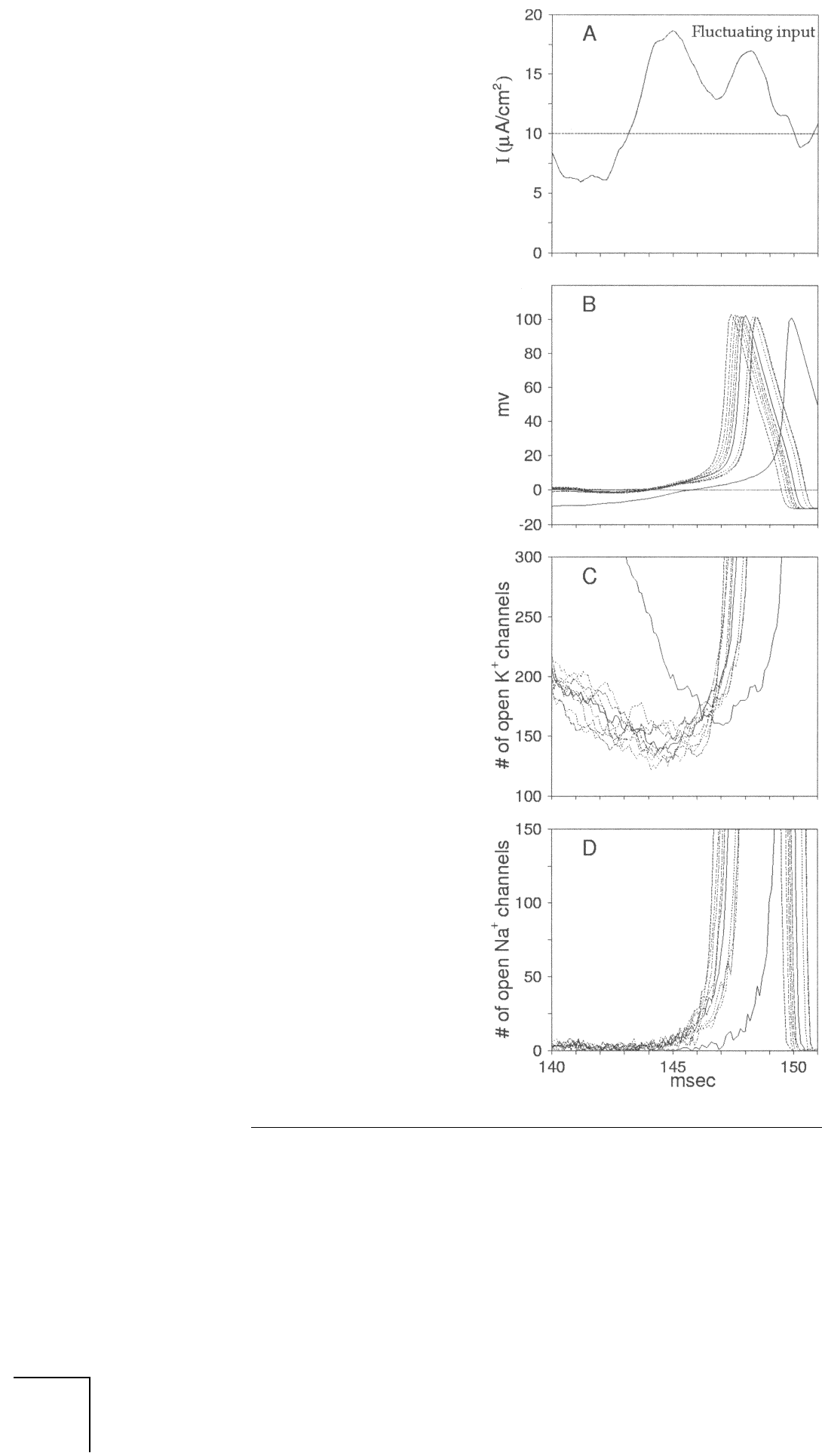
It is important to note that both the amplitude and the frequency of the membrane oscillations observed in the stochastic model are voltage dependent (e.g., compare A to B in Figure 5). This is also the case with the membrane voltage oscillations in neocortical neurons reported by Gutfreund, Yarom, and Segev (1995), Klink and Alonso (1993), as well as in other neuron types, for example, Hutcheon, Miura, Yarom, and Puil (1994) and Lampl and Yarom (1997). We suggest that in addition to the deterministic macroscopic mechanisms that were proposed to explain the generation of the subthreshold oscillations, the stochastic nature (and the limited number) of the ion channels may have a dominant effect on the nature of these oscillations (see also Longtin \& Hinzer, 1996, and Braun et al., 1998).

Channel stochasticity has such a dramatic effect on the voltage dynamics because it exploits a peculiar, and largely neglected, aspect of the deterministic $\mathrm{HH}$ equations: its two stable states for suprathreshold current input (see the discussion of the bistability in the HH equations in Cooley, Dodge, and Cohen, 1965, and Guttman, Lewis, and Rinzel, 1980). For a DC input, one state is the well-known repetitive firing behavior (the light trace in Figure $6 \mathrm{~A}$ ) whereas the other state is a nonfiring behavior of early damped voltage oscillations that converges to a steady voltage (see Figure 6A, dark trace). In both cases, a $7 \mu \mathrm{A} / \mathrm{cm}^{2} \mathrm{DC}$ current was injected, and the marked difference between the two curves is the result of minute perturbation in the initial conditions (see the caption of Figure 6 for details). These two different behaviors can be better appreciated in the phase plane diagram in panels $\mathrm{B}$ and $\mathrm{C}$ of Figure 6. Translating the ion conductances to the corresponding

Figure 4: Facing page. Fluctuating input current partially overrides the channel stochasticity and increases the reliability of spike timing. A small time window of the system behavior for the fluctuating input case is presented. (A) The input current with a mean value of $10 \mu \mathrm{A} / \mathrm{cm}^{2}$ (horizontal dotted line) and with $\sigma_{\text {input }}=$ $5 \mu \mathrm{A} / \mathrm{cm}^{2}$ and $\tau_{\text {input }}=1 \mathrm{msec}$, injected to a $600 \mu \mathrm{m}^{2}$ stochastic $\mathrm{HH}$ membrane patch is depicted. (B) Ten superimposed voltage-traces responses to repeated injection of the fluctuating current in A. In 9 out of 10 of the cases, a spike was fired within approximately 1 millisecond time window. (C, D) The number of open $\mathrm{Na}^{+}$and $\mathrm{K}^{+}$channels, respectively, for the voltage traces presented in $\mathrm{B}$, reflecting how the fluctuations of both $\mathrm{Na}^{+}$and $\mathrm{K}^{+}$channels are overridden by the fluctuations in the input current. When a sufficient number of $\mathrm{K}^{+}$channels close (C), the depolarizing transient in the input current, starting at $t=143 \mathrm{msec}$, results in the nearly synchronous buildup of $\mathrm{Na}^{+}$channels at $t=146 \mathrm{msec}(\mathrm{D})$. The result is spike firing at $t=147.3-148.4 \mathrm{msec}$. In the one case where an insufficient number of $\mathrm{K}^{+}$channels was closed in time, the spike is initiated somewhat later due to the next fluctuation in the input current. 

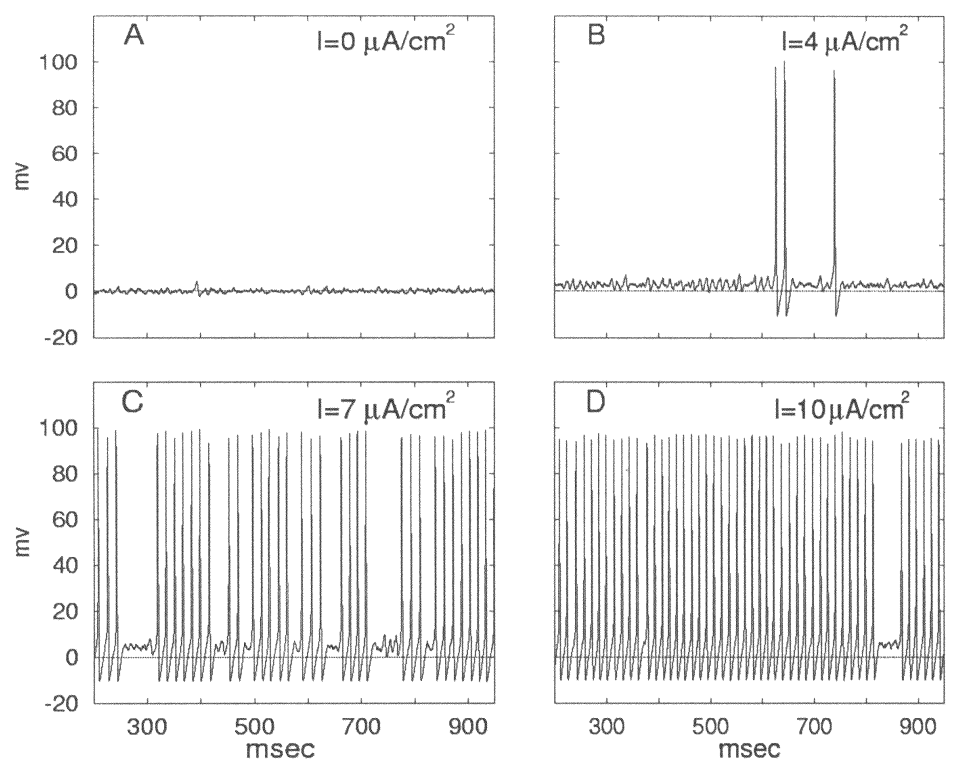

Figure 5: The response of the stochastic model to injected DC input currents. DC currents of different amplitudes were injected to a stochastic $\mathrm{HH}$ model of an isopotential membrane patch of area $600 \mu \mathrm{m}^{2}\left(10,800 \mathrm{~K}^{+}\right.$channels and $36,000 \mathrm{Na}^{+}$channels). (A, B) Membrane voltage oscillations are the dominant effect of the stochastic nature of the ion channels, with occasional spontaneous spiking. (C, D) Suprathreshold DC input currents result with irregular spiking, occasional "missing" spikes and membrane voltage oscillations. This is not expected in the corresponding deterministic $\mathrm{HH}$ model where the threshold is $7 \mu \mathrm{A} / \mathrm{cm}^{2}$. Below this value, smooth subthreshold voltage response is observed in the deterministic model (not shown); above this value, regular firing is obtained (not shown).

number of open ion channels, these panels show the very different paths in phase space taken by the firing (light curve) and the nonfiring (dark curve) trajectories. The bottom panel shows the convergence of the nonfiring behavior to a fixed point. It also shows that the distance, in terms of number of open channels, between the continuous firing cycle and the nonfiring voltage behavior is very small. Although small, the deterministic nature of the $\mathrm{HH}$ equations implies that for a DC input, the system remains in one stable state or the other. However, introduction of channel noise could, in principle, flip the system between these two states. 
Figures 6D-F show that in the stochastic model, channel fluctuations occasionally bridge the small distance in phase space between the two stable states. The stochastic opening (or closing) of a few extra $\mathrm{K}^{+}$and/or $\mathrm{Na}^{+}$ channels pushes the system spontaneously from the continuous firing stable state (light line) to the nonfiring stable state (dark line), where it stays for a while, and vice versa (at $t=772 \mathrm{msec}$ in Figure 6D). This spontaneous transition between the two states is the cause for the "missing" spikes and the subthreshold membrane voltage as well as for occasional "spontaneous spikes" (see Figure 5B). Panels E and F depict the corresponding phaseplane behavior of the system. It clearly shows that fluctuations due to only
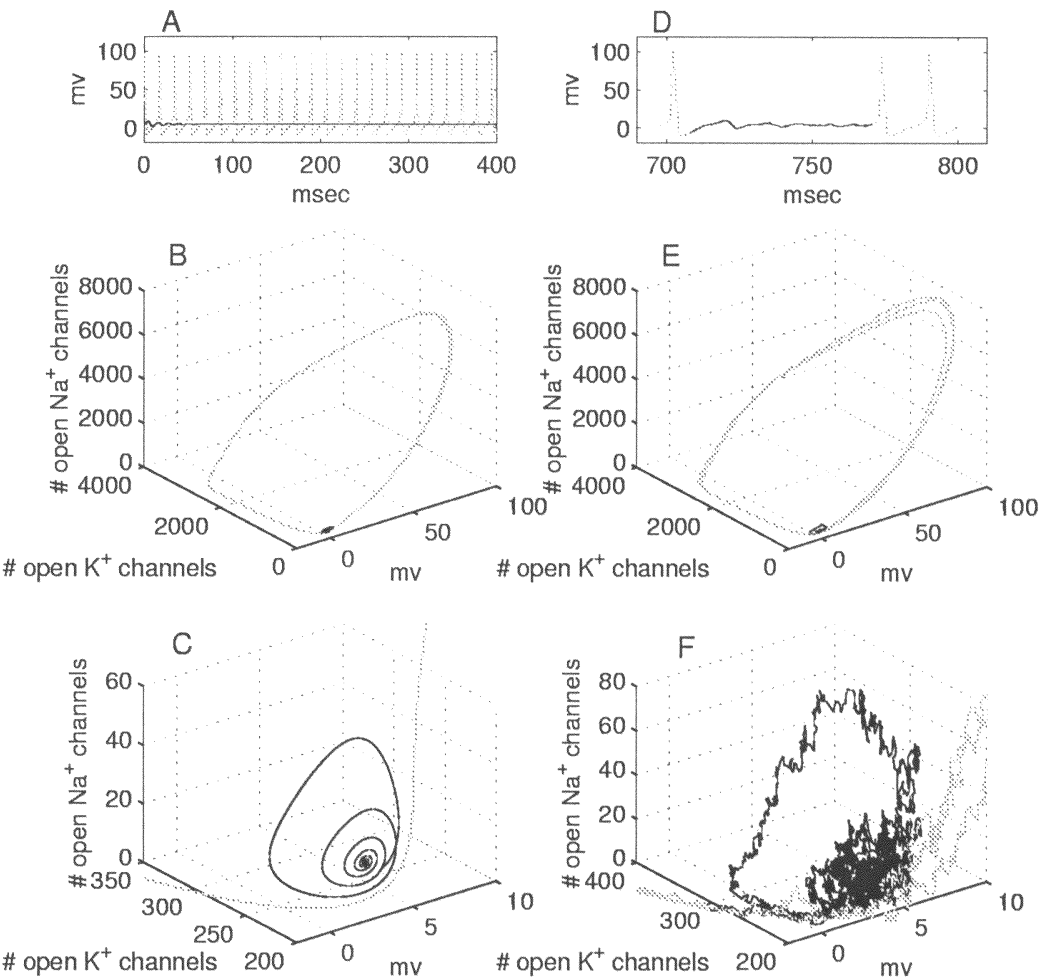

\# open $\mathrm{K}^{+}$channels 200 
a few channels are responsible for the transition between these two stable states. We conclude that the nonfiring stable state in the deterministic $\mathrm{HH}$ model becomes a key player in the stochastic HH model. Experimentally, the coexistence of the two stable solutions in the the squid giant axon, as well as in the corresponding $\mathrm{HH}$ model, was demonstrated by Guttman et al. (1980) (see also Cooley et al., 1965).

Considering the subthreshold membrane oscillations, the role of channel fluctuations is twofold. First, they drive the system from the firing state into the basin of attraction of the nonfiring stable state. Second, the fluctuations prevent the system from converging into the fixed point of the nonfiring stable state of the corresponding deterministic model. As a result, the system is cycling around this fixed point, and the subthreshold membrane voltage oscillations thus emerge. The frequency of the subthreshold oscillations is set by the period of these cycles. Based on this observation, we can predict analytically the power spectrum of the oscillations with a fair degree of accuracy, and we can also quantify the rate of transfer between the two states in the case of DC input (unpublished observations). Questions regard-

Figure 6: Previous page. Channel fluctuations cause flipping between firing and nonfiring stable states in the stochastic HH model. (A-C) The two stable states of the deterministic $\mathrm{HH}$ model. In $\mathrm{A}$, two traces of the membrane potential are shown for a $600 \mu \mathrm{m}^{2}$ membrane patch, injected with a $7 \mu \mathrm{A} / \mathrm{cm}^{2}$ DC current. The difference between the light and the dark traces results from the minute difference in the initial conditions. In the continuous firing case (light trace), the initial values are: $V=4.21 \mathrm{mV} ; g_{K}=4121 \mathrm{pS}$ (corresponds to 206.05 open $\mathrm{K}^{+}$channels) and $g_{\mathrm{Na}}=195.8 \mathrm{pS}$ (corresponding to 9.79 open $\mathrm{Na}^{+}$channels) assuming a single-channel conductance of $20 \mathrm{pS}$ (Table 1). In the nonfiring case (dark trace) the initial values are: $V=4.23 \mathrm{mV} ; g_{K}=4399.4 \mathrm{pS}$ (corresponds to 219.97 open $\mathrm{K}^{+}$channels) and $g_{\mathrm{Na}}=197.4 \mathrm{pS}$ (corresponding to 9.87 open $\mathrm{Na}^{+}$ channels). (B) The 3D phase plane of these two behaviors of the system. The light curve is for the spiking behavior, and the dark curve is for the nonfiring stable state. A magnification of B is presented in C, reflecting the small basin of attraction of the nonfiring state and the short distance in terms of number of open channels between the two states. (D-F) The corresponding behavior of $\mathrm{A}-\mathrm{C}$ in the stochastic model. Channel fluctuations in the stochastic model spontaneously flip the system between the firing and the nonfiring states. (D) a typical voltage trace of the stochastic $\mathrm{HH}$ patch. Light and dark lines were used to emphasize the different segments of the trace. The corresponding phase-plane traces are shown in E and F. As can be seen in F, the system flips from the firing stable state (light trace) to the nonfiring stable state (dark trace), where it stays for a few cycles. Hence, the subthreshold oscillations in the top trace translate to small-size loops in the phase-plane. The system then flips back to the firing stable state (light trace). 
ing the effect of various parameters on the subthreshold oscillations, such as the area of the membrane patch and the properties of the channels, will be addressed briefly in section 4 , but a more complete study is yet to be performed.

\section{Discussion}

4.1 Stochastic Versus Deterministic HH Model. We have shown that with a realistically large number of ion channels, the inherent noise in channel operation critically determines the timing and dynamics of spike firings for the stochastic $\mathrm{HH}$ model. The reason for this strong effect of channel stochasticity is that near the threshold for spike firing, only a very small percentage of $\mathrm{Na}^{+}$and a small percentage of $\mathrm{K}^{+}$channels is open (the activation variables, $m$ and $n$, are small near threshold). Consequently, the variability in membrane voltage near threshold for excitation is large, and this is reflected in the variability of spike firing time. We conclude that for a wide range of input parameters, the stochastic model captures important features of real neurons; these features are neglected in the deterministic model.

In agreement with the experimental results Mainen and Sejnowski (1995) and Nowak et al. (1997), the reliability and precision of spike timing in the stochastic $\mathrm{HH}$ model are very sensitive to the properties of the current input. The reliability and precision of the spike timing are high for strongly fluctuating inputs and decreases for smoother (e.g., DC) inputs. This study shows that this effect could be explained in terms of the relation between the instantaneous shape and amplitude of the input signal and the amplitude of channel fluctuations. Strongly fluctuating inputs "override" the inherent channel fluctuations, and the spike timing is dictated primarily by the input rather than by channel stochasticity. In contrast, channel fluctuations become relatively more significant for smooth inputs, and spike firing time becomes less reliable.

In addition to its effect of spike timing, channel stochasticity produces three additional phenomena that do not occur in the deterministic $\mathrm{HH}$ model but were all observed experimentally (e.g., see Guttman et al., 1980). Voltage membrane oscillations are seen for subthreshold current inputs, and they also occur between spikes for suprathreshold inputs. A detailed study of the amplitude and frequency of these voltage-dependent oscillations will be performed elsewhere. "Spontaneous" spikes (for subthreshold inputs) and "missing" spikes (for suprathreshold inputs) were also observed in this model. These three phenomena result from the "unmasking" of the nonfiring stable state in the $\mathrm{HH}$ model by the channel fluctuations. This state, which was largely neglected in the framework of the deterministic $\mathrm{HH}$ model, becomes a key player in determining voltage dynamics in the stochastic model. 
4.2 Toward a More Realistic Stochastic Model of Neurons. This study gives only a qualitative explanation for the reliability behaviors of neurons, in particular, of neocortical pyramidal cells. First, the spatial domain of neurons was completely neglected. It is especially important to consider the filtering effect and the impedance load imposed by the soma and dendrites, as well as by the axon, on the excitable channels at the spike initiation zone. A multicompartmental model (possibly composed of an axon with several highly excitable nodes of Ranvier, separated by passive internodes and a few dendritic compartments) should be used to understand better the effect of channel stochasticity on the reliability and accuracy of spiking in neurons. In such a model, the input should impinge onto the dendritic compartments and be simulated by a barrage of synaptic conductance changes (rather than by current inputs). In this context, it is important to emphasize that in many neuron types, the dendritic membrane is endowed with excitable channels in low density, and this may imply a large variability (fluctuations) already in the receptive region of the neuron. In contrast, we expect that in the axon, most of the variability will arise in the compartment where the spikes are initiated and that, downstream along the axon, spike timing would be encoded very reliably and with high precision. ${ }^{1}$ Still, complete failure may occur in axons at regions with a low safety factor for propagation (e.g., see Grossman, Parnas, \& Spira, 1979).

The other severe simplification in this study is that it relies exclusively on the standard $\mathrm{HH}$ equations. Although important as a reference, one ought to inquire to what extent the results can be generalized to other excitable systems. After all, most neurons consist of a large variety of ion channel types (e.g., A current, persistent and slow-inactivating $\mathrm{Na}^{+}$currents, low threshold $\mathrm{Ca}^{+2}$ current) each with different density and kinetics. Moreover, based on direct measurements of single ion channels, different kinetic models could be assigned to each of the channel types (see Patlak, 1991; Vandenberg \& Bezanilla, 1991; Marom, Salman, Lyakhov, \& Braun, 1996) although their main features are usually similar. Indeed, a more realistic model of the spike-generating mechanism that takes into account various channel types, kinetic schemes, and voltage dependence, as well as long-term (memory) effects, should be explored (see Vandenberg \& Bezanilla, 1991; Sigworth, 1993;

\footnotetext{
${ }^{1}$ One might wonder if the uncertainty engendered by the bistability in the $\mathrm{HH}$ model would make spike propagation along the axon impossible. If, at each site in the axon, there is some probability that the system will go into a stable nonfiring state, the spike may fail somewhere along the axon. Also, a significant noise in the axon (see Rubinstein, 1995) may destroy temporal correlations between the output synapses. However, except for the compartment where the spike may, or may not, be initiated as a result of the depolarizing synaptic current, all other axonal compartments downstream receive relatively sharp and large current input from the spike in the previous node. For such inputs, channel stochasticity will be masked, and, consequently, the axon is expected to act as a highly reliable delay line, adding only relatively small jitter (see Horikawa, 1991, 1993; Abeles \& Lass, 1975; Lass \& Abeles, 1975).
} 
Toib, Lyakhov, \& Marom, 1998; Abbott, Turrigiano, LeMasson, \& Marder, 1996; Fleidervish, Friedman, \& Gutnick, 1996).

Still, we can state with confidence that the surprisingly large effect of channel stochasticity is likely to persist for other models. The important parameter that determines the size of fluctuations near threshold for spike firing is the number of open channels in this voltage regime. To the best of our knowledge, in all existing models of excitability, only a small percentage of the total number of excitable channels is open near threshold. Consequently, a large variability in spike firing time is also expected in these models. Clearly the exact nature of spike firing reliability will depend on channel properties.

What about the subthreshold membrane oscillations, spontaneous spikes, and missing spikes? The nature of the bistability of the $\mathrm{HH}$ model, which is set by its inverted-Hopf bifurcation, is what "enables" the channel noise to switch the system spontaneously between its two stable states. This is the source of the subthreshold membrane oscillations, the "missing" and the "spontaneous" spikes. These phenomena may not occur in models with different types of stabilities (e.g., those with saddle-node bifurcation), and other phenomena may then arise (see Rinzel \& Ermentrout, 1989; White, Budde, \& Kay, 1995; Longtin \& Hinzer, 1996). Indeed, our initial simulations of the spike initiation zone in a cortical pyramidal neurons using parameters taken from Mainen et al. (1995) and Rapp et al. (1996) better replicate the responses of cortical pyramidal cells to the different stimuli given in Mainen and Sejnowski (1995) and Nowak et al. (1997).

4.3 Sources of Noise in Neurons. In this study we considered only the effect of one source of noise in neurons: the intrinsic stochastic nature of the ion channels. A variety of other sources of noise exists, such as spontaneous synaptic release and variability in the number of transmitter molecules and the number of available receptors. Other possible sources of neuronal noise are changes in intracellular and extracellular ion concentrations and in the concentration of neuromodulators, as well as in the activity of ion pumps. Ephaptic interactions (electric field effect) of one neuron on other neurons is yet another possible source of noise.

Our study shows that the intrinsic channel stochasticity should be considered as a key source of the variability of action potential timing. Clearly the other possible sources of noise should also be considered in order to quantify the relative contribution of each of these sources or their possible synergistic effect. Experimental studies should be performed to clarify this issue by using different manipulations-for example, blocking synaptic receptors (as did Mainen \& Sejnowski, 1995), using a dynamic clamp to "replace" the noisy channel conductance with a deterministic conductance (Sharp, O'Neil, Abbott, \& Marder, 1993), blocking specific ion channels and observing the resultant changes in membrane noise under voltage-clamp conditions (see initial results in this direction by Volgushev et al., 1998), 
and blocking ion pumps. From a theoretical viewpoint, as shown by Jensen and Gartner (1997), a simple additive noise could qualitatively reproduce the differences in reliability and accuracy of spike timing in response to DC versus the fluctuating input found experimentally. However, because the neuronal noise is both voltage and activity dependent, it is clear that a simple additive noise is only a first-order approximation to the real case. The biophysically inspired model of channel noise is inherently voltage and activity dependent. The difference between models with simple additive noise and models with more realistic noise in terms of the fine temporal structure of spike firing requires further exploration.

4.4 Implication for Neural Coding. The reliability and accuracy of the neuron firing, in both the stochastic $\mathrm{HH}$ model and in cortical neurons (Mainen \& Sejnowski, 1995; Bair \& Koch, 1996), as well as in other neurons (de Ruyter van Steveninck et al., 1997), range between an unreliable response to DC inputs and a very reliable response to large-amplitude, highly fluctuating inputs. The actual current that reaches the site of spike initiation in neurons varies between these two extreme input patterns; its exact nature is determined by the degree of correlation among the synaptic inputs that impinge onto the neuron. Highly correlated synaptic activity gives rise to sharp current transients, whereas uncorrelated synaptic activity gives rise to smoother current traces. Our modeling results suggest that the neuron's most basic machinery - the ion channels — enables it to act as a "smart" encoder. Slowly varying inputs are coded with low reliability and accuracy, and, hence, the information about such inputs is encoded almost exclusively by the spike rate. Trying to decode information about such an input, using the exact temporal structure of the spike train, would result in decoding the internal noise of the cell rather than decoding the input. On the other hand, correlated inputs are encoded with higher reliability and accuracy, giving more of a "temporal" code, that is, information about the input exists in the exact timing of the spikes.

It is clear that in such a system, correlated activity of a population of neurons is likely to propagate within the network with high temporal precision, as suggested by Abeles (1991) in his "synfire" model and by the recent work by Marsalek, Koch, \& Maunsell, (1997). In contrast, weakly correlated activity would propagate in an imprecise temporal manner and is more likely to decay within the network. The fact that the intrinsic noise of neurons may serve as a mechanism to destroy propagation of random correlations and, at the same time, allow for an accurate chains of activity to persist within the network has no bearing on the question of whether such chains do exist. Still, it is tempting to hypothesize that neurons do use their intrinsic channel stochasticity to exploit temporal code in addition to using rate code (see Bialek, Rieke, de Ruyter van Steveninck, \& Warland, 1991; de Ruyter van Steveninck et al., 1997; Hopfield, 1995; Theunissen \& Miller, 1995; Abbott, 1994; Softky, 1995; Heller, Hertz, Kjaer, \& Richmond, 1995). 
In addition to its significance for information coding, the relatively small size of the channel pool in the spike initiation zone has further computational implications. One clear advantage of such a limited channel pool was demonstrated in the work of Toib et al. (1998) (see also Marom, 1998), which shows that channel inactivation and reactivation kinetics have a significant, long-lasting (minutes) effect on the "availability" of channels, providing the neuron with an effective memory. Thus, the output spike train depends on both the properties of the instantaneous synaptic input and the history of the presynaptic and postsynaptic activity. This memory is embedded in the distribution of channel states in the spike initiation site. The nature and resolution of this memory depend on the size of the channel pool and on the kinetics and number of states of the channels. We hypothesize that the number of channels in the spike initiation zone may be optimized in some sense to give the reliability and accuracy discussed above, together with a short-term memory of the neuron's activity. In this context, it is interesting to mention the work of Marder, Abbott, Turrigiano, Liu, and Golowasch (1996) and Abbott et al. (1996), which demonstrates activity-dependent long-term changes in the properties of intrinsic membrane currents.

Another important effect of stochasticity in a limited pool of channels are the subthreshold and suprathreshold membrane oscillations. Such oscillations were observed in neocortical neurons (see Gutfreund et al., 1995) as well as in other neuron types (Hutcheon et al., 1994; Lampl \& Yarom, 1997) and were suggested to serve as the underlying clock for neurons firing and even as a synchronizing and binding mechanism for neuronal activity (Hopfield, 1995; Volgushev et al., 1997). In the stochastic HH model, these voltage oscillations result from the channel noise; in other systems, other mechanisms may be responsible for these oscillations (e.g., Gutfreund et al., 1995; White et al., 1995).

Finally, we suggest that channel stochasticity is likely to be a key player in setting neurons' firing patterns, and thus it should be incorporated in models that explore the firing variability and spike timing of cortical neurons. It seems that channel stochasticity would be dominant in models that assume balanced excitation-inhibition (see Shadlen \& Newsome, 1994, 1995; Softky, 1995; Bell, Mainen, Tsodyks, \& Sejnowski, 1995), in which the effective resting membrane voltage of the cell is near threshold. Bell et al. (1995) and Troyer and Miller (1997) suggest complex repolarization and refractoriness schemes as another source for the high firing variability in neocortical neurons, which in many ways coincide with the effect of channel fluctuations. Whatever the correct model might be, the main message of this study is that the noise inherent in the activity of ion channels must be considered if one wishes to understand what determines the firing patterns of neurons and, consequently, the nature of the neural code. 


\section{Acknowledgments}

We are grateful to our friends and colleagues, Miki London, Yosi Yarom, John Rinzel, David Hansel, Shimon Marom, Zach Mainen, Henry Markram, Tali Tishby, and Moshe Abeles, for their insightful input during various stages of this work. This study was supported by grants from the ONR, the Human Frontiers, and the Israel Academy of Science.

\section{References}

Abbott, L. (1994). Decoding neuronal firing and modeling neural networks. Quarter. Rev. Biophys., 27, 291-331.

Abbott, L., Turrigiano, G., LeMasson, G., \& Marder, E. (1996). Activitydependent conductances in model and biological neurons. In D. Waltz (Ed.), Natural and artificial parallel computing (pp. 43-68). Philadelphia: SIAM.

Abeles, M. (1991). Corticonics: Neural circuits of the cerebral cortex. Cambridge: Cambridge University Press.

Abeles, M., \& Lass, Y. (1975). Transmission of information by the axon: II. The channels capacity. Biol. Cybern., 19, 121-125.

Bair, W., \& Koch, C. (1996). Temporal precision of spike trains in extrastriate cortex of the behaving macaque monkey. Neural Comp., 8, 1185-1202.

Bell, A., Mainen, Z., Tsodyks, M., \& Sejnowski, T. (1995). "Balancing" of conductances may explain irregular cortical spiking (Tech. Rep. No. INC-9502). San Diego: Institute for Neural Computation, University of California at San Diego.

Berzukov, S., \& Vodyanoy, I. (1995). Noise-induced enhancement of signal transduction across voltage-dependent ion channels. Nature, 378, 362-364.

Bialek, W., Rieke, F., de Ruyter van Steveninck, R., \& Warland, D. (1991). Reading a neural code. Science, 252, 1854-1857.

Braun, H., Huber, M., Dewald, M., Schafer, K., \& Voigt, K. (1998). Computer simulations of neuronal signal transduction: The role of nonlinear dynamics and noise. Int. J. Bifurc. Chaos, in press.

Chow, C., \& White, J. (1996). Spontaneous action potentials due to channel fluctuations. Biophys. J., 71, 3013-3021.

Clay, J., \& DeFelice, L. (1983). Relationship between membrane excitability and single channel open-close kinetics. Biophys. J., 42, 151-157.

Cooley, J., Dodge, F., \& Cohen, H. (1965). Digital computer solutions for excitable membrane models. J. Cell. Comp. Physiol., 66, 99-100.

de Ruyter van Steveninck, R., Lewen, G., Strong, S., Koberle, R., \& Bialek, W. (1997). Reproducibility and variability in neural spike trains. Science, 275, 1805-1808.

DeFelice, L., \& Isaac, A. (1992). Chaotic states in random world. J. Stat. Phys., 70, 339-352.

Fitzhugh, R. (1965). A kinetic model of the conductance changes in nerve membrane. J. Cell. Comp. Physiol., 66, 111-118.

Fleidervish, I., Friedman, A., \& Gutnick, M. (1996). Slow inactivation of $\mathrm{Na}^{+}$ 
current and slow cumulative spike adaptation in mouse and guinea-pig neocortical neurons in slices. J. Physiol., 493, 83-97.

Grossman, Y., Parnas, I., \& Spira, M. (1979). Differential conduction block in branches of a bifurcating axon. J. Physiol., 295, 283-305.

Gutfreund, Y., Yarom, Y., \& Segev, I. (1995). Subthreshold oscillations and resonant frequency in guinea-pig cortical neurons: Physiology and modeling. $J$. Physiol., 483, 621-640.

Guttman, R., Lewis, S., \& Rinzel, J. (1980). Control of repetitive firing in squid axon membrane as a model for a neuroneoscillator. J. Physiol., 305, 377-395.

Heller, J., Hertz, J., Kjaer, T., \& Richmond, B. (1995). Information flow and temporal coding in primate pattern vision. J. Comput. Neurosci., 2, 175-193.

Hille, B. (1992) Ionic channels of excitable membrane (2nd ed.). Sunderland, MA: Sinauer Associates.

Hodgkin, A., \& Huxley, A. (1952). A quantative description of membrane current and its application to conduction and excitation in nerve. J. Physiol. (London), $117,500-544$.

Hopfield, J. (1995). Pattern recognition computation using action potential timing for stimulus representation. Nature, 376, 33-36.

Horikawa, H. (1991). Noise effects on spike propagation in the stochastic Hodgkin Huxley modles. Biol. Cybern. 66, 19-30.

Horikawa, H., (1993). Simulation study on effects of channel noise on differential conduction at an axon branch. Biophys. J., 65, 680-686.

Hutcheon, B., Miura, R., Yarom, Y., \& Puil, E. (1994). Low threshold calcium current and resonance in thalamic neurons: A model of frequency preference. J. Neurophysiol., 71, 583-594.

Jensen, R., \& Gartner, D. (1997). Synchronization of randomly driven nonlinear oscillators and the reliable firing of cortical neurons. Paper presented at the Computational Neuroscience Meeting, Big Sky, MT.

Klink, R., \& Alonso, A. (1993). Ionic mechanisms for the subthreshold oscillations and differential electroresponsiveness of medical enthorinal cortex layer II neurons. J. Neurophysiol., 70, 144-157.

Lampl, I., \& Yarom, Y. (1997). Subthreshold oscillations and resonant behavior: Two manifestations of the same mechanism. Neuroscience, 78 (2), 325-341.

Lass, Y., \& Abeles, M. (1975). Transmission of information by the axon: I. Noise and memory in the myelinated nerve fiber of the frog. Biol. Cybern., 19, 61-67.

Lecar, H., \& Nossal, R. (1971a). Theory of threshold fluctuations in nerves. I. Relationships between electrical noise and fluctuations in axon firing. Biophys. J., 11, 1048-1067.

Lecar, H. \& Nossal, R. (1971b). Theory of fluctuations in nerves. II. Analysis of various sources of membrane noise. Biophys. J., 11, 1068-1084.

Longtin, A., \& Hinzer, K. (1996). Encoding with bursting, subthreshold oscillations, and noise in mammalian cold receptors. Neural Comp., 8, 215-255.

Mainen, Z., Joerges, J., Huguenard, J., \& Sejnowski, T. (1995). A model of spike initiation in neocortical pyramidal neurons. Neuron, 15, 1427-1439.

Mainen, Z. F., \& Sejnowski, T. (1995). Reliability of spike timing in neocortical neurons. Science, 268, 1503-1508. 
Marder, E., Abbott, L., Turrigiano, G., Liu, Z., \& Golowasch, J. (1996). Memory from the dynamics of intrinsic membrane currents. Proc. Natl. Acad. Sci. USA, 93, 13481-13486.

Marom, S. (1998). Slow changes in the availability of voltage-gated ion channels: Effects on the dynamics of excitable membranes. J. Mem. Biol., 161, 105-113.

Marom, S., Salman, H., Lyakhov, V., \& Braun, E. (1996). Effects of density and gating delayed-rectifier potassium channels on resting membranes potential and its fluctuations. J. Membrane Biol., 154, 267-274.

Marsalek, P., Koch, C., \& Maunsell, J. (1997). On the relationship between synaptic input and spike output jitter in individual neurons. Proc. Natl. Acad. Sci. USA, 94, 735-740.

Nowak, L., Sanches-Vives, M., \& McCormick, D. (1997). Influence of low and high frequency inputs on spike timing in visual cortical neurons. Cerebral Cortex, 7, 487-501.

Patlak, J. (1991). Molecular kinetics of voltage-dependent $\mathrm{Na}^{+}$channels. Physiological Rev., 71(4), 1047-1080.

Press, W., Teukolsky, S., Vetterling, W., \& Flannery, B. (1992). Numerical recipes in $C$ : The art of scientific computing (2nd ed.). Cambridge: Cambridge University Press.

Rapp, M., Yarom, Y., \& Segev, I. (1996). Modeling back propagating action potential in weakly excitable dendrites of neocortical pyramidal cells. Proc. Natl. Acad. Sci. USA, 93, 11985-11990.

Reich, D., Victor, J., Knight, B., Ozaki, T., \& Kaplan, E. (1997). Response variability and timing precision of neuronal spike trains in vivo. J. Neurophysiol., 77, 2836-2841.

Rinzel, J., \& Ermentrout, B. (1989). Analysis of neural excitability and oscillations. In C. Koch \& I. Segev (Eds.), Methods in neuronal modeling (pp. 135-169). Cambridge, MA: MIT Press.

Rubinstein, J. (1995). Threshold fluctuations in an N sodium channel model of the node of ranvier. Biophys. J., 68, 779-785.

Sakmann, B., \& Neher, E. (1995). Single-channel recording (2nd ed.). New York: Plenum.

Schneidman, E., Freedman, B., \& Segev, I. (1997). Spike timing reliability in a stochastic Hodgkin-Huxley model. Paper presented at the Computational Neuroscience Meeting, Big Sky, MT.

Shadlen, M., \& Newsome, W. (1994). Noise, neural codes and cortical organization. Curr. Opin. Neurobiol., 4, 569-579.

Shadlen, M., \& Newsome, W. (1995). Is there a signal in the noise? Curr. Opin. Neurobiol., 5, 248-250.

Sharp, A., O'Neil, M, Abbott, L, \& Marder, E. (1993). The dynamic champ: Artificial conductances in biological neurons. Trends in Neurosci, 16, 389-394.

Sigworth, F. (1993). Voltage gating of ion channels. Quarter. Rev. Biophys., 27, $1-40$.

Skaugen, E., \& Walløe, L. (1979). Firing behavior in a stochastic nerve membrane model based upon the Hodgkin-Huxley equations. Acta Physiol. Scand., 107, 343-363. 
Softky, W. (1995). Simple codes versus efficient codes. Curr. Opin. Neurobiol., 5, 239-247.

Strassberg, A., \& DeFelice, L. (1993). Limitations of the Hodgkin-Huxley formalism: Effects of single channel kinetics on transmembane voltage dynamics. Neural Computation, 5, 843-856.

Tang, A., Bartels, A., \& Sejnowski, T. (1997). Cholinergic modulation preserves spike timing under physiologically realistic fluctuating input. In M. C. Mozer, M. I. Jordan, \& T. Petsche (Eds.), Advances in neural information processing systems, 9 (pp. 866-872). Cambridge, MA: MIT Press.

Theunissen, F., \& Miller, J. (1995). Temporal encoding in nervous systems: A rigorous definition. J. Comp. Neurosci., 2, 149-162.

Toib, A., Lyakhov, V., \& Marom, S. (1998). Interaction between duration of activity and rate of recovery from slow inactivation in mammalian brain $\mathrm{Na}^{+}$ channels. J. Neurosci., 18, 1893-1903.

Troyer, T., \& Miller, K. (1997). Physiological gain leads to high ISI variability in a simple model of a cortical regular spiking cell. Neural Computation, 9. 733-745.

Vandenberg, C., \& Bezanilla, F. (1991). A sodium channel gating model based on single channel, macroscopic ionic, and gating currents in the squid axon. Biophys. J., 60, 1511-1533.

Volgushev, M., Christiakova, M., \& Singer, W. (1998). Modification of discharge patterns of neocortical neurons by induced oscillations of the membrane potential. Neuroscience, 83, 15-25.

White, J., Budde, T., \& Kay, A. (1995). A bifurcation analysis of neuronal subthreshold oscillations. Biophys. J., 69, 1203-1217.

Yamada, W., Koch, C., \& Adams, P. (1989). Multiple channels and calcium dynamics. in C. Koch \& I. Segev (Eds.), Methods in neuronal modeling (pp. 97134). Cambridge, MA: MIT Press.

Received September 4, 1997; accepted January 12, 1998. 